\title{
Localized edge states in two-dimensional topological insulators: Ultrathin Bi films
}

\author{
M. Wada, ${ }^{1}$ S. Murakami,${ }^{1,2}$ F. Freimuth, ${ }^{3}$ and G. Bihlmayer ${ }^{3}$ \\ ${ }^{1}$ Department of Physics, Tokyo Institute of Technology, Ookayama, Meguro-ku, Tokyo 152-8551, Japan \\ ${ }^{2}$ PRESTO, Japan Science and Technology Agency (JST), Kawaguchi, Saitama 332-0012, Japan \\ ${ }^{3}$ Institut für Festkörperforschung and Institute for Advanced Simulation, Forschungszentrum Jülich, D-52425 Jülich, Germany
}

(Received 18 February 2011; published 21 March 2011)

\begin{abstract}
We theoretically study the generic behavior of the penetration depth of the edge states in two-dimensional quantum spin Hall systems. We found that the momentum-space width of the edge-state dispersion scales with the inverse of the penetration depth. As an example of well-localized edge states, we take the $\mathrm{Bi}(111)$ ultrathin film. Its edge states are found to extend almost over the whole Brillouin zone. Correspondingly, the bismuth (111) 1-bilayer system is proposed to have well-localized edge states in contrast to the HgTe quantum well.
\end{abstract}

DOI: 10.1103/PhysRevB.83.121310

PACS number(s): 73.43.-f, 72.25.Dc, 73.20.At, 85.75.-d

\section{INTRODUCTION}

The quantum spin Hall (QSH) phase ${ }^{1,2}$ is a new state of matter predicted theoretically, and has received a lot of attention recently. This phase is a nonmagnetic insulator in the bulk or film, and has gapless surface or edge states. The edge states consist of counterpropagating states with opposite spins. The notable feature of these edge states is that they are topologically protected; they remain gapless even in the presence of nonmagnetic impurities and interaction. ${ }^{3,4}$ We still know few systems in which the QSH phase is realized. A theoretical proposal for the QSH system on the Bi ultrathin film was made by one of the authors. ${ }^{5}$ In addition, the $\mathrm{HgTe}$ quantum well has been theoretically proposed ${ }^{6}$ and experimentally shown to be in the two-dimensional (2D) QSH state. ${ }^{7,8}$

The edge states are localized near the edge, but their penetration depth $\ell$ into the bulk varies among the systems. Observation and control of the edge states crucially depends on the penetration depth, and how they are determined in various systems is an important issue. In this Rapid Communication, we study the behavior of the penetration depth $\ell$ in QSH systems. From a simple model, we establish a simple formula for the penetration depth $\ell$. From the formula, we show that the minimum penetration depth (which is typically reached in the middle of the bulk gap) scales with the inverse of the extension of the edge states in $k$ space. Hence, if the edge states exist in a small region in $k$ space, $\ell$ is long. By generalizing this conclusion, we expect that $\ell$ is of the order of the lattice constant if the edge state extends almost over the whole Brillouin zone (BZ). To see this, we numerically study bismuth thin films and their edge states. Among bismuth thin films, only two thin films are proposed to be insulating in the bulk: the (111) single- (1-) bilayer film ${ }^{9}$ and the $\{012\}$ 2-monolayer film. ${ }^{10}$ By using tight-binding (TB) Hamiltonians obtained by first-principles calculations, we find that (111) 1-bilayer film is in the QSH phase and $\{012\}$ 2-monolayer film is not. We also find that the edge states in $\mathrm{Bi}$ (111) 1-bilayer film are well localized near the edges, compared with the HgTe quantum well. From these studies, we conclude that the penetration depth $\ell$ corresponds to the inverse of the $k$-space width of edge-state dispersion. We note that the penetration depth $\ell$ was studied in Refs. 11 and 12, but for a ribbon geometry, where it is difficult to derive an analytic formula for $\ell$. In contrast, our calculation on a semi-infinite plane enables us to derive the analytic formula for $\ell$ and discuss a wide range of systems.

\section{PENETRATION DEPTH OF THE EDGE STATES}

We use the Hamiltonian for the HgTe quantum well

$$
\mathcal{H}\left(k_{x}, k_{y}\right)=\left(\begin{array}{cc}
H(\mathbf{k}) & 0 \\
0 & H^{*}(-\mathbf{k})
\end{array}\right),
$$

where $H(\mathbf{k})=\epsilon_{\mathbf{k}} \mathbf{I}_{2}+d^{a}(\mathbf{k}) \sigma^{a}$. Here, $\mathbf{I}_{2}$ is a $2 \times 2$ unit matrix, $\sigma_{a}$ are the Pauli matrices, $\epsilon_{\mathbf{k}}=C-D\left(k_{x}^{2}+k_{y}^{2}\right), d^{1}=A k_{x}$, $d^{2}=A k_{y}$, and $d^{3}=\mathcal{M}(k)=M-B\left(k_{x}^{2}+k_{y}^{2}\right)$. The overall energy offset $C$ is set to zero. The eigenenergies are then given by $-D k^{2} \pm|\mathbf{d}(\mathbf{k})|$. Thus, $D$ represents the asymmetry between the valence and the conduction band dispersions. The bulk gap at $\mathbf{k}=0$ is $2 M$. To consider the edge state on a single edge, we deal with a system on a half-plane of $y \leqslant 0$. This considerably simplifies the results, compared with the ribbon of finite width. ${ }^{11}$ As edge states, only the solutions with $e^{\lambda y}$ $(\operatorname{Re} \lambda>0)$ are allowed. The secular equation

$$
\begin{aligned}
& {\left[M-E+B_{+}\left(\lambda^{2}-k_{x}^{2}\right)\right]\left[-M-E-B_{-}\left(\lambda^{2}-k_{x}^{2}\right)\right]} \\
& \quad=A^{2}\left(k_{x}^{2}-\lambda^{2}\right),
\end{aligned}
$$

where $B_{ \pm}=B \pm D$ gives two allowed values for $\lambda$ :

$$
\lambda=\lambda_{1,2}=\sqrt{k_{x}^{2}+F \pm \sqrt{F^{2}-\left(M^{2}-E^{2}\right) /\left(B_{+} B_{-}\right)}},
$$

where $F=\frac{A^{2}-2(M B+E D)}{2 B_{+} B_{-}}$. If we impose a boundary condition $\psi(y=0)=0$ as in Ref. 11, we get

$$
\lambda_{1} \lambda_{2}=\frac{B M+D E}{B_{+} B_{-}}-k_{x}^{2}, \quad \lambda_{1}+\lambda_{2}=\frac{D M+B E}{k_{x} B_{+} B_{-}} .
$$

We then obtain an exact form for the dispersion of edge states

$$
E=\left(-D M \pm A \sqrt{B_{+} B_{-}} k_{x}\right) / B
$$

from Eqs. (3) and (4). The signs correspond to the two branches of edge states with opposite spins. Because they are related with each other by the Kramers theorem, we henceforth consider only the plus sign in (5). Putting (5) into (4), we get

$$
\lambda_{1} \lambda_{2}=-k_{x}^{2}+\frac{2 D N}{B} k_{x}+\frac{M}{B}, \quad \lambda_{1}+\lambda_{2}=2 N,
$$



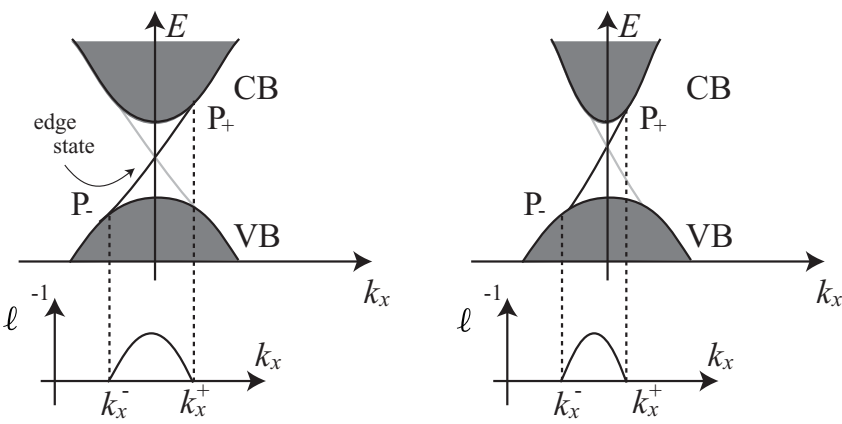

FIG. 1. Penetration depth $\ell$ for the effective model with ribbon geometry. CB (VB) represents the bulk conduction (valence) band. The plot on the right corresponds to a more asymmetric situation, leading to a larger $\ell$ at the crossing point of the edge states.

where $N=A /\left(2 \sqrt{B_{+} B_{-}}\right)$. These determine $\lambda_{1,2}$. If we put $\lambda_{1}>\lambda_{2}, \lambda_{2}^{-1}$ gives the physical penetration depth $\ell$ as discussed in Ref. 11. At the points with $\lambda_{2}=0$, the edge states have infinite penetration depth and become bulk states. From (6), this occurs when $k_{x}=k_{x}^{ \pm} \equiv \frac{D N}{B}\left(1 \pm \sqrt{1+\frac{B M}{D^{2} N^{2}}}\right)$. It can be checked that the states at $k_{x}=k_{x}^{ \pm}$are located at the band edge of the projection of the bulk band and, at these points, the edge dispersion (5) is tangential to the bulk band projection. We can rewrite as $\lambda_{1} \lambda_{2}=-\left(k_{x}-k_{x}^{+}\right)\left(k_{x}-k_{x}^{-}\right)$. Therefore, $\lambda_{2}\left(=\ell^{-1}\right)$ is expressed as

$$
\ell^{-1}=N-\sqrt{N^{2}+\left(k_{x}-k_{x}^{+}\right)\left(k_{x}-k_{x}^{-}\right)} .
$$

Hence, the behavior of $\ell^{-1}$ is as shown in Fig. 1. It vanishes at the points $P_{ \pm}\left(k_{x}=k_{x}^{ \pm}\right)$, where the edge states are absorbed into the bulk band, and $\ell$ is minimum when $k_{x}=\left(k_{x}^{+}+k_{x}^{-}\right) / 2$. The minimum value $\ell_{\min }$ is given by $\ell_{\min }^{-1}=$ $N-\sqrt{N^{2}-\left(k_{x}^{+}-k_{x}^{-}\right)^{2} / 4}$. As a function of $N$, the minimum value of $\ell_{\min }$ is $2 /\left(k_{x}^{+}-k_{x}^{-}\right)$at $N=\left(k_{x}^{+}-k_{x}^{-}\right) / 2$. This means that the minimum $\ell_{\min }$ of the system is roughly given by the inverse of the $k$-space extension of the edge-state dispersion. From Fig. 1, it can be seen that the penetration depth $\ell$ becomes short when the considered edge state is far from the points $P_{ \pm}$. The inverse of the penetration depth $\ell^{-1}$ corresponds to an imaginary part of the wave number perpendicular to the edge direction and it therefore behaves similarly to the (real) wave number. Hence, $\ell^{-1}$ is approximately given by the $k$-space distance of wave numbers from the points $P_{ \pm}$.

In the HgTe quantum wells, the 2D quantum spin Hall states are confirmed by transport measurements. ${ }^{7,8}$ The penetration depth of the edge states in these systems has been calculated to be relatively long, $\ell \sim 50 \mathrm{~nm} .{ }^{11}$ In our theory, by plugging the parameters into our results, we get $\ell=56 \mathrm{~nm}$ at $k_{x}=0$, in agreement with Ref. 11. The coefficient $D$ gives rise to an asymmetry between the conduction and the valence bands, and the edge state is also asymmetric: $k_{x}^{+} \neq-k_{x}^{-}, k_{x}^{+}=$ $0.62 \mathrm{~nm}^{-1}, k_{x}^{-}=-0.024 \mathrm{~nm}^{-1}$. Thus, the penetration depth $\ell$ is shortest not at $k_{x}=0$ but at $k_{x}=\left(k_{x}^{+}+k_{x}^{-}\right) / 2=0.30 \mathrm{~nm}^{-1}$ with $\ell_{\min } \sim 6.2 \mathrm{~nm}$. In our interpretation, the relatively long $\ell$ of the edge states in the HgTe quantum well comes from the fact that the edge states are localized within a very narrow region in $k$ space. This penetration depth determines the minimal width of the system required for observation of edge states.

We note that the present solution for the semi-infinite plane also gives physical properties for the states on the ribbon discussed in Ref. 11. When the ribbon is wider than $\ell$, the hybridization between the two edge states at the opposite edges can be treated within first-order perturbation. This hybridization induces the gap, which is an exponentially decreasing function of the ribbon width. The case with a very narrow ribbon is beyond the scope of this paper because it is far from the topological insulator.

\section{Bi(111) ULTRATHIN FILM}

By extending our theory to generic types of edge states, we can expect that the inverse of the penetration depth $\ell^{-1}$ well scales with the $k$-space distance from the absorption point $P_{ \pm}$ into the bulk band. Therefore, if the edge states extend over the Brillouin zone, the penetration depth of the edge states is as short as a few lattice constants. We will theoretically show that $\mathrm{Bi}(111)$ ultrathin film is a QSH system having edge states with such a short penetration depth.

For the calculation, we use a TB model constructed from maximally localized Wannier orbitals ${ }^{13}$ obtained from firstprinciples calculations. ${ }^{14}$ The Fermi energy lies in the six $p$-like states, comprising three conduction bands and three valence bands. Therefore, in constructing the Wannier orbitals, we only retain these six bands. From these Wannier orbitals, including the lattice relaxation effects of the ultrathin films, we construct TB models keeping up to third-neighbor hopping amplitudes.

Figures 2(a)-2(c) show the unit cell and lattice vectors, reciprocal vectors, and the time-reversal-invariant momenta (TRIM) and the energy band of $\mathrm{Bi}(111)$ 1-bilayer, respec-
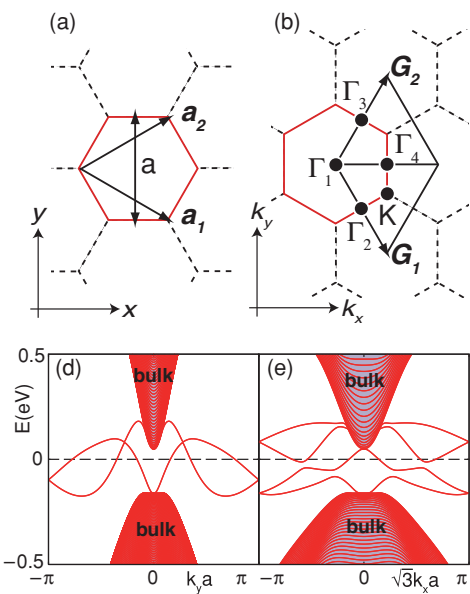
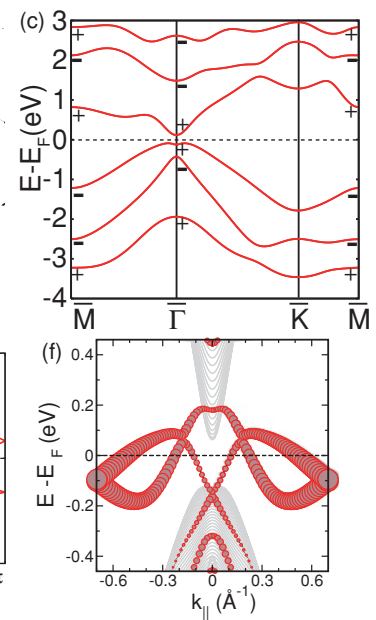

FIG. 2. (Color online) (a) Unit cell and lattice vectors and (b) TRIMs of $\mathrm{Bi}(111)$ ultrathin film. The TRIMs consist of the $\Gamma$ point and the three $M$ points. (c) Bulk energy bands and the parity at the TRIMs for a Bi(111) 1-bilayer. (d) and (e): Energy bands of the $\mathrm{Bi}(111)$ zigzag- and armchair-edge ribbons, respectively, with a width of 20 unit cells, calculated from the TB model. (f) Energy bands of a eight-unit-cell-wide $\mathrm{Bi}(111)$ zigzag-edge ribbon from first-principles calculations. The size of the symbols corresponds to the weight of the states in the edge atoms. 
tively. Since this system is inversion symmetric, all states in Fig. 2(c) are doubly degenerate. This system is proposed to be a nonmagnetic insulator with a bulk gap of $0.2 \mathrm{eV}{ }^{9} \mathrm{We}$ will calculate the $Z_{2}$ topological number $v$. For inversionsymmetric systems, $(-1)^{\nu}$ is equal to the product of all the parity eigenvalues of the Kramers pairs of eigenstates at all the TRIMs below the Fermi energy. ${ }^{15}$ The parity eigenvalues at the TRIMs are given in Fig. 2(c) and yield $v=1$. We note that the first-principles calculation (without a TB model) also gives $v=1$, meaning that the (111) 1-bilayer bismuth film is in the QSH phase, in agreement with Ref. 5 based on a simple truncation of the three-dimensional TB model. ${ }^{16}$

If we neglect the out-of-plane coordinate, the (111) 1-bilayer film has a honeycomb structure. Therefore, as in graphene, we refer to the two types of simple edge shapes as zigzag and armchair edges. Figures 2(d) and 2(e) show the energy bands of zigzag- and armchair-edge ribbons of the Bi(111) 1-bilayer. All the states are doubly degenerate, consisting of two states with opposite spins, localized on the opposite edges. In both figures, the number of Kramers pairs of edge states per one edge is odd, confirming that the $\mathrm{Bi}(111)$ 1-bilayer is a QSH system. We checked that, for the zigzag-edge ribbon, our result from the TB model [Fig. 2(d)] and that from a first-principles calculation [Fig. 2(f)] are in good agreement.

These edge states extend almost over the whole Brillouin zone. It is quite different from the HgTe quantum well, where the edge states exist only near $k=0 .{ }^{6}$ At the Fermi energy, there are three Kramers pairs of edge states. Thus, the conductance of the ribbon is $G=6 e^{2} / h$ for a clean system. When nonmagnetic disorder is increased, some of these edge states become gapped due to elastic scattering, while at least one pair of edge states remains gapless, giving $G=2 e^{2} / h$. The edge states form perfectly conducting channels, similar to those in the graphene nanoribbons. ${ }^{17}$ In graphene, perfectly conducting channels are formed only in the absence of shortranged disorder; in the $\mathrm{Bi}$ (111) 1-bilayer nanoribbon, the perfectly conducting channel exists irrespective of the nature of nonmagnetic disorder, and it gives a universal behavior realizable in experiments.

\section{Bi $\{012\}$ ULTRATHIN FILM}

For inversion-asymmetric systems such as $\mathrm{Bi}\{012\}$ 2-monolayer film, the calculation of $v$ is complicated because the phases of the Bloch wave functions in the entire BZ are involved. ${ }^{1,18}$ The phase of the wave function is a gauge degree of freedom and can be chosen arbitrary for each $\mathbf{k}$, which often causes instability in numerical calculations. Hence, we adopt a gauge-invariant discretization method proposed in Ref. 19. It is a merit of the method that we do not need to determine the phase of the wave function smoothly in $\mathbf{k}$ space. The mesh size $\delta k_{1} \delta k_{2}$ should be fine enough to satisfy $|F(\mathbf{k})| \delta k_{1} \delta k_{2}<\pi$ at any mesh, where $F(\mathbf{k})$ is the Berry curvature, and $\delta k_{1}, \delta k_{2}$ are the width and height of a mesh, respectively. ${ }^{19}$ This quantity is largest when $\mathbf{k}$ is at the direct gap $\mathbf{k}=\mathbf{k}_{\mathrm{g}}$, and the critical size is approximated by the $\mathbf{k}$-space nominal size of the band extremum at $\mathbf{k}=\mathbf{k}_{\mathrm{g}}$. From the band structure of $\operatorname{Bi}\{012\}$ 2-monolayer, the critical mesh number $n_{B}^{c}$ is estimated to be $\sim 100$. For various mesh numbers exceeding $n_{B}^{c}$, we
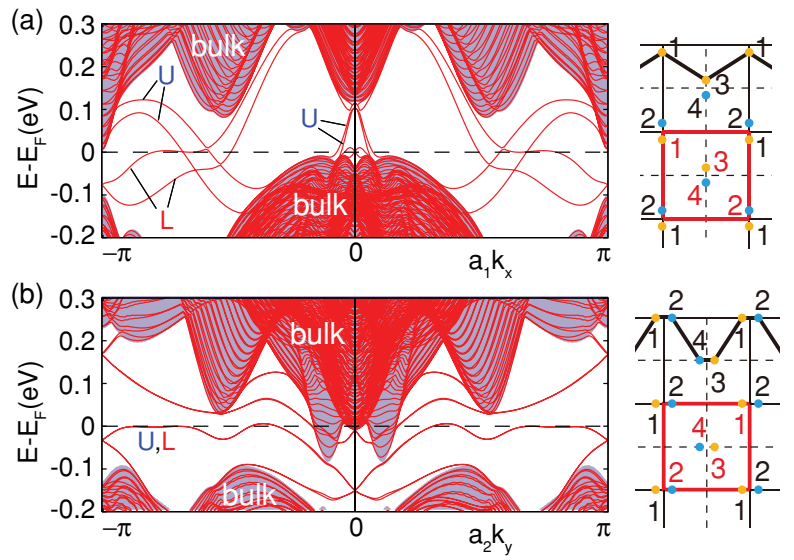

FIG. 3. (Color online) Energy bands of $\mathrm{Bi}\{012\}$ : (a) zigzag- and (b) armchair-edge ribbons with a width of 20 unit cells. $U(L)$ means that the state is localized on the upper (lower) edge. The crystal structures near the upper edge are shown in the right panels with 1 , 2, 3, and 4 representing the lattice sites. We note that these four sites do not lie on the same plane. The shaded regions are the bulk energy bands.

get $v=0$. Therefore, the $\operatorname{Bi}\{012\}$ 2-monolayer is an ordinary insulator.

The edge states of $\mathrm{Bi}\{012\}$ 2-monolayer ribbons for two types of edges are shown in Fig. 3. These two edge shapes can be called zigzag and armchair edges, although the lattice structure is different from graphene. The number of Kramers pairs of edge states on the Fermi energy is even at each edge, and this is in agreement with our result that $v=0$. The edge states of the armchair-edge ribbon are almost degenerate due to mirror symmetry, while those of the zigzag-edge ribbon are not. The small energy splitting in the edge states of the armchair-edge ribbon is due to hybridization of the edge states at the opposite edges. Nevertheless, for a ribbon wider than $\ell$, the energy splitting is exponentially small.

\section{PENETRATION DEPTH OF THE EDGE STATES}

Our calculation result of the penetration depth $\ell$ of the edge states of the zigzag-edged $\mathrm{Bi}(111)$ 1-bilayer ribbon is shown in Fig. 4(a). The penetration depths $\ell$ are typically several lattice constants. Hence, for transport experiments, the sample width has to be larger than a few lattice constants. These results on $\mathrm{Bi}(111)$ film agree with our theory on $\ell$. According to our theory, $\ell$ becomes short when the edge states are distant from the points $P_{ \pm}$where the edge states merge into the bulk [circles in Figs. 4(b) and 4(c)]. Hence, $\ell$ is longer for the states at $E_{F}$ in Fig. 4(b) and shorter in Fig. 4(c). This information is relevant for transport that is governed by the states at the Fermi level. In $\mathrm{Bi}(111)$, the edge state travels almost over the whole BZ. Therefore, we estimate $\ell \sim(\text { size of the BZ) })^{-1} \sim$ (lattice spacing), in agreement with the results in Fig. 4(a).

Bi(111) 1-bilayer film can not be described by an effective model near $\mathbf{k}=0$ such as (1). The effective model (1) is derived when the QSH system is described as a band inversion between two doubly degenerate bands, such as the HgTe 
(a)
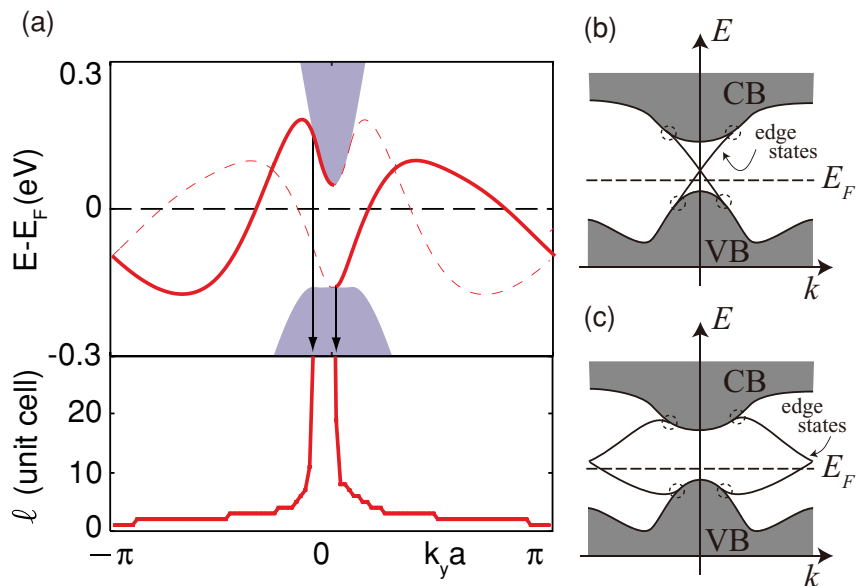

FIG. 4. (Color online) (a) Penetration depth of the edge states on the zigzag edge of the $\mathrm{Bi}(111)$. (b), (c) Examples of the edge states in the 2D QSH systems.

quantum well. In bismuth ultrathin films, the involved bands are $p_{x}, p_{y}, p_{z}$ orbitals, and the valence and conduction bands have different mixing coefficients for these orbitals. Therefore, it is not a mere band inversion, which is the reason why the case [Fig. 4(c)] is realized in bismuth films. We note that $\mathrm{Bi}_{2} \mathrm{Te}_{3}$ and $\mathrm{Bi}_{2} \mathrm{Se}_{3}$ ultrathin films also have edge states similar to Fig. 4(c). ${ }^{20}$ Although the penetration depth is not discussed in Ref. 20, it might be interesting to check whether our theory holds also in these films. We note that our theory assumes isotropy between the direction along the edge (surface) and that perpendicular to it. For layered materials such as $\mathrm{Bi}_{2} \mathrm{Se}_{3}$ and $\mathrm{Bi}_{2} \mathrm{Te}_{3}$, the penetration depth perpendicular to the layer can not be predicted from the surface-state dispersion in the layer because of the anisotropy.

These short penetration depths of edge states in the $\mathrm{Bi}$ (111) 1-bilayer film are ideal for observation by scanning tunneling microscopy and scanning tunneling spectroscopy and control of the edge states. Furthermore, it is also favorable for edge thermoelectric transport. ${ }^{21}$ To utilize the perfectly conducting channels of edge states for thermoelectric transport, short penetration depth is an important factor, because longer penetration depth mixes the states at different edges for narrow ribbons and destroys the coherent edge transport.

\section{CONCLUSION}

We derived a general and simple formula (7) for the penetration depth of the edge states in two-dimensional quantum spin Hall systems. We found that momentum-space distance between the edge states and the absorption point of the edge dispersion into the bulk band roughly gives the inverse of the penetration depth. As an example, we calculated the penetration depth of the edge states of $\mathrm{Bi}(111)$ 1-bilayer film, which we propose to be a QSH insulator. The penetration depth of the edge states in $\mathrm{Bi}(111)$ 1-bilayer film is in good agreement with our theory.

\section{ACKNOWLEDGMENTS}

We are grateful to S. Blügel, T. Hirahara, T. Nagao, and S. Yaginuma for helpful discussions. This research was supported in part by MEXT KAKENHI (Grants No. 21000004 and No. 22540327).
${ }^{1}$ C. L. Kane and E. J. Mele, Phys. Rev. Lett. 95, 226801 (2005); 95, 146802 (2005).

${ }^{2}$ B. A. Bernevig and S.-C. Zhang, Phys. Rev. Lett. 96, 106802 (2006). ${ }^{3}$ C. Wu, B. A. Bernevig, and S.-C. Zhang, Phys. Rev. Lett. 96, 106401 (2006).

${ }^{4}$ C. Xu and J. E. Moore, Phys. Rev. B 73, 045322 (2006).

${ }^{5}$ S. Murakami, Phys. Rev. Lett. 97, 236805 (2006).

${ }^{6}$ B. A. Bernevig, T. L. Hughes, and S.-C. Zhang, Science 314, 1757 (2006).

${ }^{7}$ M. König et al., Science 318, 766 (2007).

${ }^{8}$ A. Roth et al., Science 325, 294 (2009).

${ }^{9}$ Yu. M. Koroteev, G. Bihlmayer, E. V. Chulkov, and S. Blügel, Phys. Rev. B 77, 045428 (2008).

${ }^{10}$ S. Yaginuma et al., J. Phys. Soc. Jpn. 77, 014701 (2008).

${ }^{11}$ B. Zhou, H.-Z. Lu, R.-L. Chu, S.-Q. Shen, and Q. Niu, Phys. Rev. Lett. 101, 246807 (2008).
${ }^{12}$ R.-L. Chu, J. Li, J. K. Jain, and S.-Q. Shen, Phys. Rev. B 80, 081102(R) (2009).

${ }^{13}$ N. Marzari and D. Vanderbilt, Phys. Rev. B 56, 12847 (1997).

${ }^{14}$ F. Freimuth, Y. Mokrousov, D. Wortmann, S. Heinze, and S. Blügel, Phys. Rev. B 78, 035120 (2008).

${ }^{15}$ L. Fu and C. L. Kane, Phys. Rev. B 76, 045302 (2007).

${ }^{16}$ Y. Liu and R. E. Allen, Phys. Rev. B 52, 1566 (1995).

${ }^{17}$ K. Wakabayashi, Y. Takane, and M. Sigrist, Phys. Rev. Lett. 99, 036601 (2007)

${ }^{18}$ L. Fu and C. L. Kane, Phys. Rev. B 74, 195312 (2006).

${ }^{19}$ T. Fukui and Y. Hatsugai, J. Phys. Soc. Jpn. 76, 053702 (2007).

${ }^{20}$ C.-X. Liu, H. Zhang, B. Yan, X.-L. Qi, T. Frauenheim, X. Dai, Z. Fang, and S.-C. Zhang, Phys. Rev. B 81, 041307(R) (2010).

${ }^{21}$ R. Takahashi and S. Murakami, Phys. Rev. B 81, 161302(R) (2010). 